\title{
Onset of Vibrational Convection in a Binary Fluid Saturated Non-Darcy Porous Layer Heated from Above
}

\author{
T. Sivakumar ${ }^{\mathrm{a}}$ and S. Saravanan \\ Department of Mathematics, Bharathiar University, Coimbatore 641 046, Tamil Nadu, India
}

\begin{abstract}
A linear stability analysis is used to investigate the influence of mechanical vibration on the onset of thermosolutal convection in a horizontal porous layer heated and salted from above. Vibrations are considered with arbitrary amplitude and frequency. The Brinkman extended Darcy model is used to describe the flow and the Oberbeck-Boussinesq approximation is employed. Continued fraction method and Floquet theory are used to determine the convective instability threshold. It is found that the solutal Rayleigh number has the stabilizing effect. The existence of a closed disconnected loop of synchronous mode is predicted in the marginal curve for moderate values of solutal Rayleigh number and vibration amplitude.
\end{abstract}

\section{Introduction}

The study of thermal convection in a fluid layer with modulated gravitational field is an important class of problem in heat transfer. The effect of gravity modulation on a convectively stable configuration can significantly influence the stability of a system by increasing or decreasing its susceptibility to convection. Gresho and Sani [1] and Gershuni et al. [2] have first investigated the e ffect of sinusoidal gravity modulation in a differentially heated fluid layer through linear stability analysis. They found that the system is stabilized for small amplitude vertical oscillations. These results were later confirmed by Biringen and Peltier [3] who made a more general nonlinear three dimensional analysis. Aniss et al. [4] studied the influence of time dependent gravity on the instability threshold for a fluid layer confined in a Hele-Shaw geometry. They found that the parametric oscillations can affect the convective instability threshold when the Prandtl number is small compared to unity. The first experimental study on Rayleigh-Bénard convection in the presence of vertical vibration was conducted by Rogers et al. [5]. Their interest was in the study of the complex ordered patterns of convection generated under such conditions. These and all other related studies found in the literature involving thermal convection with time dependent gravity were all motivated by the difficulties faced during crystal growth, solidification of molten alloys and other related industrial processes.

The studies dealing with gravity modulation on convection in porous media induced by mechanical vibrations are quite recent. Zenkovskaya and Rogovenko [6] and Govender [7] have investigated the effect of vertical vibration on the onset of convection in a Darcian porous layer subject to high frequency and low-amplitude vibrations respectively. Recently, Strong [8] reported the results of this problem for arbitrary range of modulation parameters by the use of continued fraction method. There are also few recent works available in the literature dealing with thermovi-

\footnotetext{
a e-mail: thirumalaisiva@gmail.com
}

brational convection in a more general Brinkman porous medium (see [9-12]). In our recent study [9], the stability of convection in a Brinkman porous layer in the presence of vibrations of arbitrary amplitude and frequency was considered. It was demonstrated that the vibration amplitude favors as well as suppresses the setting up of convection depending on the vibration frequency for a layer heated from below whereas it always favors convection irrespective of the vibration frequency for a layer heated from above.

Convection occurring in fluids when spatial variations of a salt, with a different molecular diffusivity, is added to the existing thermal gradients is termed as double diffusive convection or more specifically thermosolutal convection. The double diffusive convection of the HortonRogers-Lapwood problem for various thermal and solutal boundary conditions was first investigated by Nield [13] using linear stability theory. Tanton et al. [14] extended Nield's analysis and considered fingering convection in a porous layer. Rudraiah et al. [15] performed finite amplitude study based on a truncated representation of Fourier series and found that subcritical instabilities are possible in the case of two component fluids. Poulikakos [16] extended the study to Lapwood-Brinkman case and presented the critical limits for both monotonic and oscillatory modes. The growing volume of work devoted to this area with different orientations of thermal and solutal gradients with respect to each other are well documented by Nield and Bejan [17].

There are also few works available in the literature pertaining to the stability of thermovibrational convection in a binary fluid saturated porous medium. The influence of mechanical vibration on the onset of convection in a confined cavity saturated with two-component fluid was examined by Jounet and Bardan [18]. It was shown that, when the solutal and thermal buoyancy forces are opposing, there is a possibility of Hopf bifurcation. A brief review of the findings related to the above subject have been given by Razi et. al [19]. Recently, Strong [20] investigated the vi- 
brational thermosolutal convection in a horizontal Darcy porous layer and constructed the neutral curves for synchronous, subharmonic and quasiperiodic instability modes. More recently, Siddheshwar et al [21] performed a weakly nonlinear analysis for this problem using Ginzburg-Landau equation in the presence of small amplitude oscillations. In the present study, our aim is to investigate the effect of vertical harmonic vibration on the onset of thermosolutal convection for a wide range of modulation parameters in a Brinkman porous medium heated from above.

\section{Mathematical formulation}

We consider a binary fluid saturated porous layer, confined between two horizontal surfaces $z=0$ and $z=h$ of infinite extent. The layer is both heated and salted from above. We assume that the layer and its boundaries are subjected to vertical harmonic vibration. Brinkman's law is used to model the flow through it after neglecting inertial effects. The equation of state has

$$
\rho=\rho_{0}\left[1-\beta\left(T-T_{0}\right)+\beta_{c}\left(C-C_{0}\right)\right]
$$

where $\rho$ is the density, $T$ the temperature, $C$ the concentration, $\beta$ the thermal expansion coefficient and $\beta_{c}$ the concentration expansion coefficient $\left(\beta>0\right.$ and $\left.\beta_{c}>0\right)$. The appropriate equations governing laminar flow through the porous medium under the Oberbeck-Boussinesq approximation are

$$
\begin{gathered}
\frac{1}{\varphi} \frac{\partial \mathbf{v}}{\partial t}+\frac{1}{\varphi^{2}} \mathbf{v} \cdot \nabla \mathbf{v}=-\frac{1}{\rho} \nabla p-\frac{v}{K} \mathbf{v}+\nu \nabla^{2} \mathbf{v}+\left(\beta T-\beta_{c} C\right) g(t) \widehat{\mathbf{k}} \\
\varkappa \frac{\partial T}{\partial t}+\mathbf{v} \cdot \nabla T=\chi \nabla^{2} T \\
\varphi \frac{\partial C}{\partial t}+\mathbf{v} \cdot \nabla C=D_{m} \nabla^{2} C \\
\nabla \cdot \mathbf{v}=0
\end{gathered}
$$

where $\mathbf{v}=\left(v_{1}, v_{2}, v_{3}\right)$ is the filtration velocity, $p$ the pressure, $\varphi$ the porosity, $K$ the permeability, $v$ the kinematic viscosity, $\widehat{\mathbf{k}}$ the unit vector directed vertically upward, $x$ the heat capacity ratio, $\chi$ the thermal diffusivity and $D_{m}$ the mass diffusivity of the porous medium. Time dependent gravitational field is taken to be $g(t)=g_{0}+\frac{A}{\varphi} \Omega^{2} f^{\prime \prime}(\tau)$, where $g_{0}$ is a reference acceleration level, $A$ the vibration amplitude, $\Omega$ the vibration frequency and $f(\tau)$ the $2 \pi$ periodic function with zero $2 \pi$-average.

We study the stability of the following quiescent basic state $\mathbf{v}^{0}=0, T^{0}=T_{1}-a z$ and $C^{0}=C_{1}-b z$, where $a=\left(T_{2}-T_{1}\right) / h$ and $b=\left(C_{2}-C_{1}\right) / h$, using the method of small perturbations. The following scales are used to nondimensionalize the variables:

$$
(x, t, \mathbf{v}, p, T, C) \rightarrow\left(h, \frac{h^{2}}{v}, \frac{v}{h}, \frac{\rho v^{2}}{K}, a h, b h\right)
$$

The non-dimensional governing equations are

$$
\begin{array}{rl}
c \frac{\partial \mathbf{u}}{\partial t}=-\nabla q-\mathbf{u}+D & a \nabla^{2} \mathbf{u}+\left(G r_{T} \theta-G r_{S} \phi\right)\left(1+\eta f^{\prime \prime}(\tau)\right) \widehat{\mathbf{k}} \\
\varkappa \frac{\partial \theta}{\partial t}-u_{3} & =\frac{1}{P r} \nabla^{2} \theta \\
\varphi \frac{\partial \phi}{\partial t}-u_{3} & =\frac{1}{S c} \nabla^{2} \phi \\
\nabla \cdot \mathbf{u} & =0
\end{array}
$$

where $D a=K / h^{2}$ the Darcy number, $G r_{T}=\beta a h^{2} g_{0} K / v^{2}$ the thermal Grashof number, $G r_{S}=\beta_{c} b h^{2} g_{0} K / v^{2}$ the solutal Grashof number, $\operatorname{Pr}=v / \chi$ the Prandtl number, $S c=$ $v / D_{m}$ the Schmidt number, $c=D a / \varphi$ the porosity - permeability parameter, $\eta=A \Omega^{2} / \varphi g_{0}$ the amplitude and $\omega=$ $\Omega h^{2} / v$ the frequency of modulation. The nondimensional stress free boundary conditions are

$$
u_{3}=\frac{\partial^{2} u_{3}}{\partial z^{2}}=\theta=\phi=0 \text { at } z=0 \text { and } z=1
$$

We eliminated the pressure and performed the normal mode analysis. From the resulting equations the $z$-variable is separated by taking $\left[\widetilde{u_{3}}(z, t), \widetilde{\theta}(z, t), \widetilde{\phi}(z, t)\right]=\left[\widehat{u_{3}}, \widehat{\theta}, \widehat{\phi}\right](t) \sin (\pi z)$

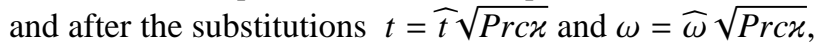
we obtain a system of ordinary differential equations with periodic coefficients:

$$
\begin{aligned}
& \left.\frac{c}{r} \frac{d \widehat{u_{3}}}{d \widehat{t}}=-\left(\operatorname{Dam}^{2}+1\right) \widehat{u_{3}}+\frac{\alpha^{2}}{m^{2}}\left(1+\eta f^{\prime \prime}(\tau)\right)\left(G r_{T} \widehat{\theta}-G r_{S} \widehat{\phi}\right) 12\right) \\
& \frac{x}{r} \frac{\widehat{d \theta}}{d \widehat{t}}=\widehat{u_{3}}-\frac{m^{2}}{P r} \widehat{\theta} \\
& \frac{\varphi}{r} \frac{\widehat{d \phi}}{d \widehat{t}}=\widehat{u_{3}}-\frac{m^{2}}{S c} \widehat{\phi}
\end{aligned}
$$

where $\alpha^{2}$ is the overall horizontal wavenumber, $m^{2}=\alpha^{2}+$ $\pi^{2}$ and $r=\sqrt{\operatorname{Prc} x}$. We assume that $f(\tau)=\cos \omega t$ in Eq.(12) and for notational convenience, tilde will be subsequently omitted.

Following the Floquet theory, we search for the solution to the system (12)-(14)in the form

$$
\left(\widehat{u_{3}}, \widehat{\theta}, \widehat{\phi}\right)(t)=e^{\sigma t} \sum_{n=-\infty}^{+\infty}\left(w_{n}, \theta_{n}, \phi_{n}\right) e^{i n \omega t}
$$

where $\sigma$ is the Floquet exponent that defines the behaviour of the perturbation with time. Substitution of Eq.(15) into the system (12)-(14) yields an infinite tridiagonal system of linear algebraic equations for determination of unknown coefficient $\theta_{n}$ :

$$
M_{n} \theta_{n}+q_{n-1} \theta_{n-1}+q_{n+1} \theta_{n+1}=0, n=\ldots,-2,-1,0,1,2,
$$

Here $R a_{T}=G r_{T} \cdot \operatorname{Pr}$ is the thermal Rayleigh number, $R a_{S}=G r_{S} \cdot S c$ is the solutal Rayleigh number, Le $=S c / P r$ is the Lewis number and $L=L e \varphi / x$.

Now we use the continued fraction method to solve the above linear system. Substituting $\zeta_{n}=\theta_{n-1} / \theta_{n},\left(\theta_{n} \neq 0\right)$, the system (16) becomes

$$
M_{n}+\left(q_{n-1} \zeta_{n}+\frac{q_{n+1}}{\zeta_{n+1}}\right)=0, n=\ldots,-2,-1,0,1,2, \ldots
$$

The validity of the transition from Eqs.(16)-(17) was discussed by Strong [8] and they proved that none of the coefficients $\theta_{n}$ can become zero for a solution of a system (16). From Eq. (17), using complex fractions, we derive two different recurrence relations for the unknown $\zeta_{n}$ which in turn yield two different continued fractions. Assigning $n=0$ in these leads to the following dispersion equation 
for the Floquet exponent $\sigma$ in the explicit form

$$
M_{0}-\frac{q_{0} q_{1}}{M_{1}-\frac{q_{1} q_{2}}{M_{2}-\frac{q_{2} q_{3}}{M_{3}-\cdots}}}=\frac{q_{0} q_{-1}}{M_{-1}-\frac{q_{-1} q_{-2}}{M_{-2}-\frac{q_{-2} q_{-3}}{M_{-3}-\cdots}}}
$$

from which we can determine the values of the Floquet exponent $\sigma$.

The Eq.(18) is simplified to the real form when $\sigma=0$ corresponding to the synchronous mode (S) with period $2 \pi / \omega$ with the symmetry $M_{-n}=\overline{M_{n}}$ and $q_{-n}=\overline{q_{n}}$ (bar denotes the complex conjugate) as

$$
\operatorname{Re}\left(\frac{q_{0} q_{1}}{M_{1}-\frac{q_{1} q_{2}}{M_{2}-\frac{q_{2} q_{3}}{M_{3}-\cdots}}}\right)=\frac{M_{0}}{2}
$$

The Eq.(18) is simplified to the real form when $\sigma=i \omega / 2$ corresponding to the subharmonic mode (SH) with period $4 \pi / \omega$ with the symmetry $M_{-n}=\overline{M_{n-1}}$ and $q_{-n}=\overline{q_{n-1}}$ as

$$
\left|M_{0}-\frac{q_{0} q_{1}}{M_{1}-\frac{q_{1} q_{2}}{M_{2}-\frac{q_{2} q_{3}}{M_{3}-\cdots}}}\right|^{2}=q_{0}^{2}
$$

The transcendental Eqs. (19) and (20) are solved then to obtain the marginal curves of $R a_{T}$ against $\alpha$ for $\mathrm{S}$ and $\mathrm{SH}$ modes respectively. Prior to that convergence of the continued fractions was verified numerically and the continued fractions were truncated once the desired precision $\left(10^{-4}\right)$ is achieved. The stability characteristics, viz., the critical Rayleigh number $R a_{T, c}$, obtained by minimizing marginal $R a_{T}$ against $\alpha$ and the critical wavenumber $\alpha_{c}$, the $\alpha$ corresponding to $R a_{T, c}$ are then calculated by fixing the values of other parameters.

\section{Results and discussion}

The effect of time-periodically varying gravity field on the onset of thermosolutal convection in a horizontal porous layer is investigated. Attention is paid to the situation in which the vibrating porous layer is both heated and soluted from above. We present the results for arbitrary values of amplitude $\eta$ and frequency $\omega$. Also we fixed $\operatorname{Pr}=1$, $\varkappa=1$ and $c=D a=0.1$ corresponding to Brinkman porous medium throughout the study. The expression (16) contains, in particular, the corresponding result of Strong [20] for $D a=0$, binary fluid case for the Darcy model and Saravanan and Sivakumar [9] for $R a_{S}=0$, pure fluid case for the Brinkman model.

The variation of $R a_{T, c}$ and $\alpha_{c}$ against $\omega$ are shown in Figs. 1 and 2, respectively, for different values of $\eta, R a_{S}$ and $L=10$. We observed that $R a_{T, c} \rightarrow \infty$ for $\omega \rightarrow \infty$ because the modulation has no effect on high frequency

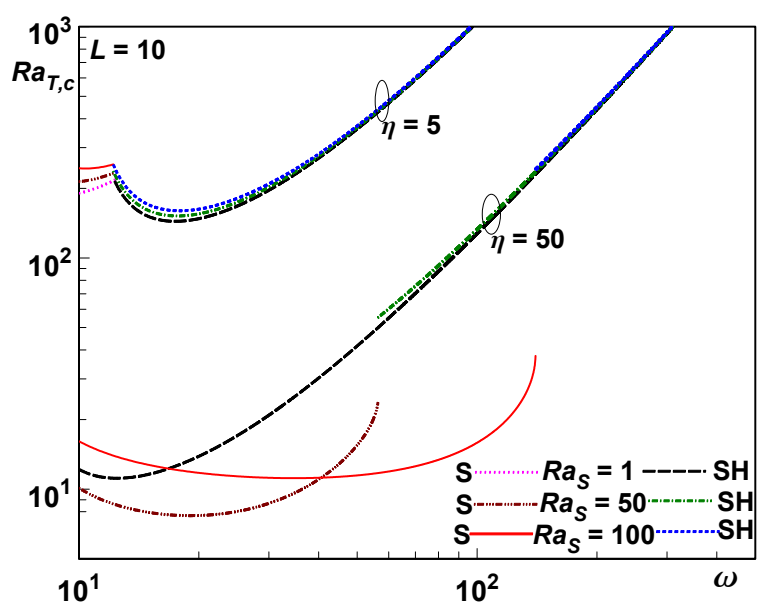

Fig. 1. $R a_{T, c}$ against $\omega$ for different $\eta, R a_{S}$ and $L=10$.

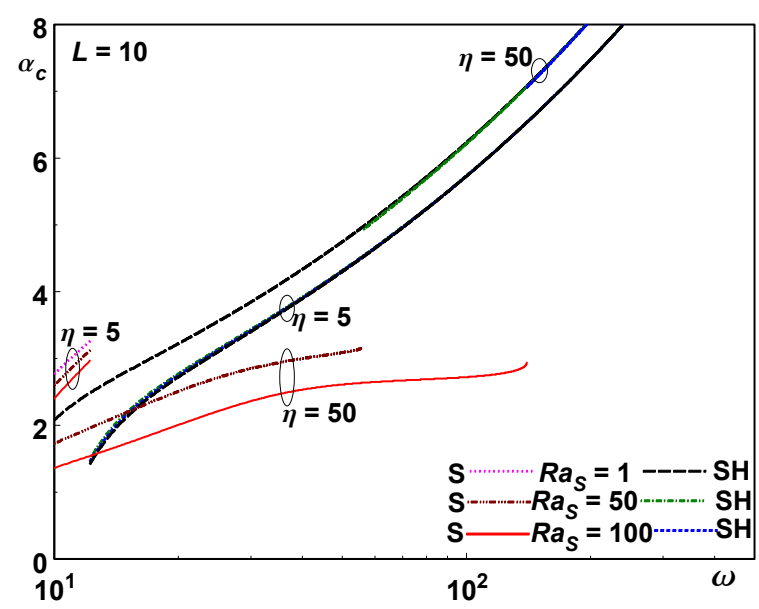

Fig. 2. $\alpha_{c}$ against $\omega$ for different $\eta, R a_{S}$ and $L=10$.

and it corresponds to the unmodulated problem. For $\eta=5$, we observe that the solutal Rayleigh number $R a_{S}$ slightly stabilizes the system for lower $\omega$ and its effect becomes insignificant for higher $\omega$. Moreover $R a_{T, c}$ exhibits a cusp at a lower $\omega$ which is due to the change of the underlying instability mode, i.e., the onset of instability is of $S$ type upto this $\omega$ and of SH type beyond this $\omega$. The corresponding $\alpha_{c}$ (Fig.2) jumps from a higher to a lower value. We now explain this transition in terms of marginal curves. The solution through the Floquet analysis revealed marginal curves in the form of a group of loop shaped branches enclosing the region of instability [9]. The bottommost loop was $\mathrm{S}$ for very small $\omega$ and the minimum of this loop determined the onset condition. An additional thin SH loop emerged and developed in the low wavenumber region for an increase in $\omega$ and at one stage its minimum reached the level of the already existing $\mathrm{S}$ loop. Hence $R a_{T, c}$ for both the $\mathrm{S}$ and $\mathrm{SH}$ modes were equal (bicritical) at the transition frequency with two different $\alpha_{c}$ values. For a further increase in $\omega$ beyond this transition frequency the $\mathrm{SH}$ mode grew further and became critical. We note that there is no mode transition beyond this cusp and the SH mode is the preferred one throughout the frequency range.

From Fig.1, we see that the effect of solutal Rayleigh number $R a_{S}$ is quite different for large amplitude $\eta=50$. For $R a_{S}=1$, the onset of instability is dictated solely by the SH mode. The stability limits exhibit an interesting be- 


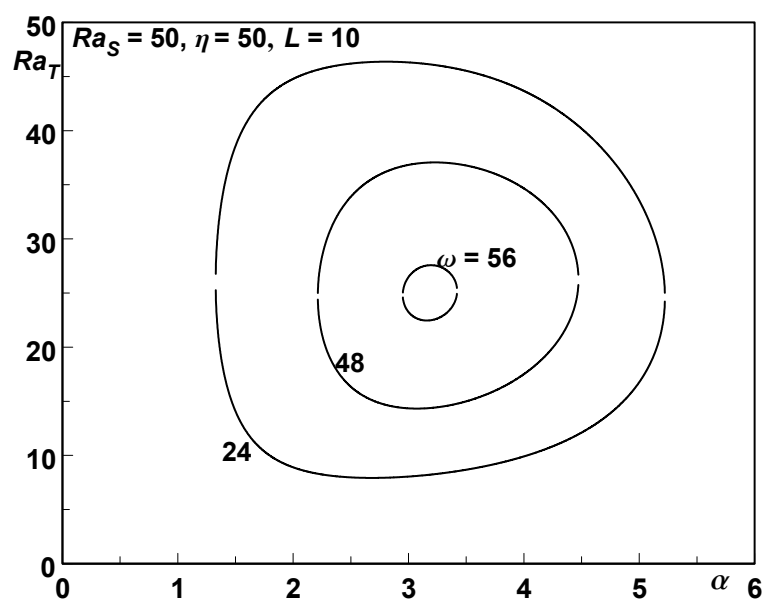

Fig. 3. $\mathrm{CDL}$ in the marginal curve with $\mathrm{S}$ resonant loops for different values of $\omega$.

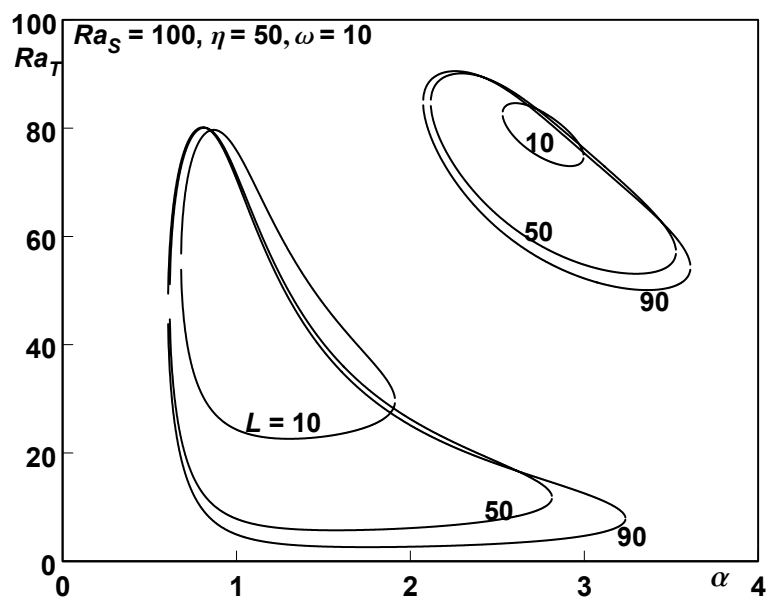

Fig. 4. CDL in the marginal curve with $\mathrm{S}$ resonant loops for different values of $L$

haviour for $R a_{S}=50$ and 100. Unlike the low amplitude case, $R a_{T, c}$ discontinuously jumps from $\mathrm{S}$ to $\mathrm{SH}$ mode at the transition frequency [11]. This is different from the bicritical situation which was discussed earlier. The changes taking place in the marginal curve in the neighbourhood of the transition frequency deserve mentioning at this stage. Figure 3 shows a set of closed disconnected loops (CDL) of $S$ type (see $[11,20]$ ) for $R a_{S}=50$ and different $\omega$. Although there exist multiple loops above each CDL in the marginal curve, they are not displayed. As $\omega$ increases from 24 to 56 , the CDL shrinks and finally disappears at the transition frequency $\omega=56.4$. Hence the corresponding $R a_{T, c}$ jumps to the already existing $\mathrm{SH}$ loop. From Fig.2, we see that $\alpha_{c}$ jumps from higher to lower values for $\eta=5$ whereas it jumps from lower to higher values for $\eta=50 . \alpha_{c}$ for $\mathrm{S}$ mode decreases and $\mathrm{SH}$ mode increases as $\eta$ takes higher values. Also we note that the jumps in $\alpha_{c}$ are small at low frequencies and they become large at high frequencies implying that the CDL forms well below the $\mathrm{SH}$ loop at low wavenumber region and determine the critical condition for high frequencies. One important observation is the existence of two CDLs in the marginal curve for $\mathrm{S}$ mode and are displayed in Fig.4 for different values of $L$. An increase in $L$ make the CDLs bigger. It is evident from this figure that $L$ destabilizes the system for the parameters under consideration. Thus in general we observe that $\eta$ favours the onset of convection for the layer heated from above. The existence of the CDL extends the S mode to higher frequency range.

\section{Conclusion}

The onset of thermovibrational convection in two component fluid saturated porous layer is investigated using the Brinkman model. The conditions for instability have been obtained via a linear stability analysis and by employing the continued fraction method with the aid of Floquet theory. The study leads to the following conclusions. The solutal Rayleigh number delays the onset condition. The existence of a CDL in the marginal curve for synchronous mode of instability is predicted for moderate values of solutal Rayleigh number and vibration amplitude.

The authors thank University Grants Commission, India for its support through DRS Special Assistance Programme in Fluid Dynamics. This work was carried out as a part of a research project (Grant No. 25(0169)/09/EMR-II) awarded by Council of Scientific and Industrial Research, India. Its financial assistance to one of the authors (T.S.) is gratefully acknowledged.

\section{References}

1. P.M. Gresho, R.L. Sani, J. Fluid Mech. 40, 783 (1970).

2. G.Z. Gershuni, E.M. Zhukhovitskii, I.S. Iurkov, J. Appl. Math. Mech. 34, 442 (1970).

3. S. Biringen, L. J. Peltier, Phys. Fluids 2, 754 (1990).

4. S. Aniss, M. Souhar, M. Belhaq, Phys. Fluids 12, 262 (2000).

5. J. Rogers, M. Schatz, J. Bougie, J. Swift, Phys. Rev. Lett. 84, 87 (2000).

6. S.M. Zen'kovskaya, T.N. Rogovenko, J. Appl. Mech. Tech. Phys. 40, 379 (1999).

7. S. Govender, Transp. Porous Media 57, 113 (2004).

8. N. Strong, J. Math. Fluid Mech. 10, 488 (2008).

9. S. Saravanan, T. Sivakumar, Phys. Fluids 22, 034104 (2010).

10. S. Saravanan, A. Arunkumar, Int. J. Eng. Sci. 48, 742 (2010).

11. S. Saravanan, T. Sivakumar, ASME J. Heat Transfer 133, 051601 (2011).

12. S. Saravanan, T. Sivakumar, Phys. Rev. E 84, 026307 (2011).

13. D.A. Nield, Water Resour. Res. 4, 553 (1968).

14. J.W. Tanton, E.N. Lighfoot, T. Green, Phys. Fluids 15, 748 (1972).

15. N. Rudraiah, P.K. Srimani, R. Friedrich, Int. J. Heat Mass Transfer 25, 715 (1982).

16. D. Poulikakos, Int. Commun. Heat Mass Transfer 13, 587 (1986).

17. D.A. Nield, A. Bejan, Convection in Porous Media (Springer-Verlog, New York, 2006).

18. A. Jounet, G. Bardan, Phys. Fluids 13, 1 (2001).

19. Y.P. Razi, A. Mojtabi, M.C. Charrier-Mojtabi, Trans. Porous Media 77, 207 (2009).

20. N. Strong, SIAM J. Appl. Math. 69, 1263 (2008).

21. P.G. Siddheshwar, B.S. Bhadauria, S. Alok, Transp. Porous Media (2011). 